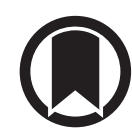

CrossMark

\title{
JAK inhibitors in COVID-19: the need for vigilance regarding increased inherent thrombotic risk
}

\author{
To the Editor:
}

There is accumulating evidence that coronavirus disease 2019 (COVID-19) is a hypercoagulable state. Reports of thrombotic events and autopsy findings of pulmonary thrombotic microangiopathy in patients with COVID-19 are rising [1]. BOMPARD et al. [2] recently reported a cohort study of 137 patients with COVID-19 pneumonia, in which retrospective review of computed tomography pulmonary angiography scans demonstrated a cumulative incidence of pulmonary emboli (PE) of $24 \%$ overall and $50 \%$ in intensive care. Although it was initially thought that insidious venous thromboembolic events (VTE) were mainly confined to ventilated patients [3], we now understand thrombotic risk to be a wider problem in COVID-19. An overexuberant host inflammatory response in selected patients with severe COVID-19 may contribute to the high mortality. We recently recommended screening for virally driven hyperinflammation in COVID-19 and proposed that immunomodulation in this subgroup of patients may improve outcomes [4]. There are several ongoing, randomised controlled trials evaluating the therapeutic potential of janus kinase inhibitors (JAKi) in severe COVID-19 (table 1). JAKi have a purported advantage over other immunomodulatory strategies in COVID-19, as they may exert dual anti-inflammatory (blockade of multiple, pro-inflammatory cytokines simultaneously) and anti-viral effects (impeding cellular viral endocytosis $[5,6]$ ) and have convenient oral administration, with relatively short half-lives. JAKi may interrupt the signalling of several pro-inflammatory cytokines implicated in the pathogenesis of hyperinflammation, including interleukin-6, which has been the focus of several clinical trials in COVID-19. JAKi may also inhibit the entry of severe acute respiratory syndrome coronavirus 2 virus into the alveolar type 2 alveolar epithelial cells; baricitinib (a JAK1/2 inhibitor) is a numb-associated kinase inhibitor, with a particularly high affinity for alveolar type 2 cell-associated protein kinase 1 , a pivotal regulator of clathrin-mediated viral endocytosis [5]. We recommend vigilance to the potentially increased thrombotic risk associated with JAKi, given the hypercoagulability of COVID-19 and our recent thromboprophylaxis recommendations for all hospitalised patients with COVID-19 [7].

Several JAKi are licenced in rheumatoid arthritis and have Food and Drug Administration (FDA) black box warnings for venous and arterial thrombotic events (including deep venous thrombosis (DVT), PE and ischaemic stroke), resulting in dose restrictions (table 1). It is unclear whether the presumed prothrombotic risks are dependent on JAK selectivity, drug specificity, dose or treatment duration or are confounded by indication. Whilst there is evidence of dose-dependent increased thrombotic risk associated with tofacitinib (five-fold increased risk of $\mathrm{PE}$ compared with tumour necrosis factor therapy in rheumatoid arthritis) [8], a recent meta-analysis did not show an overall JAKi class prothrombotic signal [9]. Although a significantly increased incidence of thrombotic events (DVT and PE) was reported with bariticitinb in rheumatoid arthritis trials [10], this has not been observed in extension studies and recent trials in atopic dermatitis [11]. Post-marketing surveillance may delineate the true risk and whether this is disease-specific. It is unclear which JAK isoform (selectivity) confers impact on the efficacy or influences the safety of these therapies for their licenced indications (e.g. rheumatoid arthritis) or in the new disease setting of COVID-19. It is important to note that at high doses, JAKi can become "pan-JAK" inhibitors and exhibit non-selectivity [12]

@ERSpublications

JAK inhibitors have promising therapeutic potential in COVID-19 with dual anti-inflammatory and anti-viral effects. Vigilance to the potentially increased thrombotic risk associated with JAKi is recommended, given the hypercoagulability of COVID-19. https://bit.ly/2NQ15K5

Cite this article as: Mehta P, Ciurtin C, Scully M, et al. JAK inhibitors in COVID-19: the need for vigilance regarding increased inherent thrombotic risk. Eur Respir J 2020; 56: 2001919 [https://doi.org/ 10.1183/13993003.01919-2020]. 
TABLE 1 Summary of relevant licensed janus kinase inhibitors ( JAKi) and thrombotic concerns

\begin{tabular}{|c|c|c|c|c|}
\hline JAKi and selectivity & $\begin{array}{l}\text { Pharmaceutical } \\
\text { company }\end{array}$ & $\begin{array}{l}\text { Licensed } \\
\text { indication }\end{array}$ & Notes regarding thrombosis & $\begin{array}{l}\text { COVID-19 trials } \\
\text { registered at } \\
\text { ClinicalTrials.gov }\end{array}$ \\
\hline JAK 1/2, ruxolitinib & Novartis & $\begin{array}{l}\text { Myelofibrosis } \\
\text { Polycythaemia }\end{array}$ & No safety alert for thrombosis & $\begin{array}{l}\text { NCT0 } 4348071 \\
\text { NCT0 } 4355793 \\
\text { NCT0 } 4354714 \\
\text { NCT0 } 4362137 \\
\text { NCT0 } 4377620 \\
\text { NCT0 } 4334044 \\
\text { NCT0 } 4337359 \\
\text { NCT04331665 } \\
\text { NCT04366232 } \\
\text { NCT04374149 } \\
\text { NCT04361903 } \\
\text { NCT04338958 } \\
\text { NCT0 } 4348695 \\
\text { NCT04359290 }\end{array}$ \\
\hline JAK $1 / 3$, tofacitinib & Pfizer & $\begin{array}{l}\text { Rheumatoid } \\
\text { arthritis } \\
\text { Psoriatic } \\
\text { arthritis } \\
\text { Ulcerative } \\
\text { colitis }\end{array}$ & $\begin{array}{l}\text { February 2019: FDA issued safety alert concerning } \\
\text { thrombotic risk } \\
\text { July 2019: FDA black box warning for DVT and PE, for the } \\
10 \mathrm{mg} \text { BD dose (this higher dose is approved for } \\
\text { ulcerative colitis) } \\
\text { November 2019: EMA updated European product } \\
\text { information with warning for thrombosis }\end{array}$ & $\begin{array}{l}\text { NCT04390061 } \\
\text { NCT04332042 }\end{array}$ \\
\hline
\end{tabular}

It is not clear whether the coronavirus disease 2019 (COVID-19) trials excluded patients at risk of thrombosis or mandated thromboprophylaxis. SmPC: summary of medicinal product characteristics; PE: pulmonary emboli; DVT: deep venous thrombosis; FDA: Food and Drug Administration; OD: once daily; BD twice daily: EMA: European Medicines Agency. ${ }^{\#}$ : n=27 as of 18 May 2020.

Discussion continues regarding whether JAKi have a causal role in thrombotic events, or whether this represents a higher background thrombotic risk [13]. Indeed, VTE risk in rheumatoid arthritis (0.3-0.7 per 100 patient years) is greater compared with the general population (0.1-0.4 per 100 patient years) [13] Intriguingly, unlike baricitinib, ruxolitinib does not carry a VTE warning, despite both being selective JAK $1 / 2$ inhibitors. Conversely, it has been suggested that ruxolitinib may lower the inherently raised thrombotic risk in myeloproliferative neoplasms [14]. Extrapolating this reassurance for ruxolitinib to COVID-19 is inappropriate as data for baricitinib and ruxolitinib are derived from different disease populations and their respective safety signals may be confounded by indication.

Clinical trials in COVID-19 using immunomodulation, including JAKi, intend to recruit patients with the most severe disease, and it is hypothesised that these patients are more hypercoagulable. We recommend risk mitigation strategies, including considering excluding patients with high thrombotic risk, treatment with standard- or intermediate-dose low molecular weight heparin prophlyaxis [7] during hospitalisation and considering extended-duration thromboprophylaxis after discharge from hospital, with monitoring for thromboses during the treatment and follow-up periods.

JAKi represent significant therapeutic advances but are relatively new drugs with evolving safety profiles. Potential prothrombotic risk may be a class effect of JAKi, which is concerning given the evidence of 
hypercoagulability in severe COVID-19. Cross-speciality communication is imperative when drug repurposing in the rapidly evolving pace of a pandemic.

Puja Mehta ${ }^{1,2}$, Coziana Ciurtin $\oplus^{2}$, Marie Scully ${ }^{3}$, Marcel Levi ${ }^{4}$ and Rachel C. Chambers $\oplus^{1}$

${ }^{1}$ Centre for Inflammation and Tissue Repair, UCL Respiratory, Division of Medicine, University College London, London, UK. ${ }^{2}$ Dept of Rheumatology, University College London (UCL) Centre for Adolescent Rheumatology, London, UK. ${ }^{3}$ Dept of Haematology, University College London Hospital (UCLH), London, UK. ${ }^{4}$ Dept of Medicine, University College London Hospitals (UCLH), London, UK.

Correspondence: Rachel C. Chambers, Centre for Inflammation and Tissue Repair, UCL Respiratory, Rayne Building, University College London, London WC1E 6JF, UK. E-mail: r.chambers@ucl.ac.uk

Received: 21 May 2020 | Accepted after revision: 27 June 2020

Author contribution: P. Mehta drafted the manuscript. All authors contributed to discussions, revised and approved the manuscript.

Conflict of interest: P. Mehta is an MRC-GSK EMINENT clinical training fellow with project funding outside the submitted work, and receives co-funding by the NIHR University College London Hospitals Biomedical Research Centre (UCLH BRC). C. Ciurtin has nothing to disclose. M. Scully has nothing to disclose. M. Levi has nothing to disclose. R.C. Chambers reports grants from UKRI MRC, GlaxoSmithKline and NIHR ULCH BRC, unrelated to this work.

\section{References}

1 Connors JM, Levy JH. COVID-19 and its implications for thrombosis and anticoagulation. Blood 2020; 135: 2033-2040.

2 Bompard F, Monnier H, Saab I, et al. Pulmonary embolism in patients with COVID-19 pneumonia. Eur Respir J 2020; 56: 2001365

3 Criel M, Falter M, Jaeken J, et al. Venous thromboembolism in SARS-CoV-2 patients: only a problem in ventilated ICU patients, or is there more to it? Eur Respir J 2020; 56: 2001201.

4 Mehta P, McAuley DF, Brown M, et al. COVID-19: consider cytokine storm syndromes and immunosuppression. Lancet 2020; 395: 1033-1034.

$5 \quad$ Stebbing J, Phelan A, Griffin I, et al. COVID-19: combining antiviral and anti-inflammatory treatments. Lancet Infect Dis 2020; 20: 400-402.

6 Richardson P, Griffin I, Tucker C, et al. Baricitinib as potential treatment for 2019-nCoV acute respiratory disease. Lancet 2020; 395: e30-e31.

7 Levi M, Thachil J, Iba T, et al. Coagulation abnormalities and thrombosis in patients with COVID-19. Lancet Haematol 2020; 7: e438-e440.

8 European Medicines Agency. Increased risk of blood clots in lungs and death with higher dose of Xeljanz (tofacitinib) for rheumatoid arthritis. https://www.ema.europa.eu/en/news/increased-risk-blood-clots-lungs-deathhigher-dose-xeljanz-tofacitinib-rheumatoid-arthritis Date last accessed: 16 May 2020. Date last updated: 20 March 2019.

9 Xie W, Huang Y, Xiao S, et al. Impact of janus kinase inhibitors on risk of cardiovascular events in patients with rheumatoid arthritis: systematic review and meta-analysis of randomised controlled trials. Ann Rheum Dis 2019; 78: 1048-1054.

10 Taylor PC, Weinblatt ME, Burmester GR, et al. Cardiovascular safety during treatment with baricitinib in rheumatoid arthritis. Arthritis Rheumatol. 2019; 71: 1042-1055.

11 Simpson EL, Lacour J-P, Spelman L, et al. Baricitinib in patients with moderate-to-severe atopic dermatitis and inadequate response to topical corticosteroids: results from two randomized monotherapy phase III trials. $\mathrm{Br} \mathrm{J}$ Dermatol 2020; 183: 242-255.

12 Winthrop KL. The emerging safety profile of JAK inhibitors in rheumatic disease. Nat Rev Rheumatol 2017; 13: 234-243.

13 Scott IC, Hider SL, Scott DL. Thromboembolism with janus kinase (JAK) inhibitors for rheumatoid arthritis: how real is the risk? Drug Saf 2018; 41: 645-653.

14 Samuelson BT, Vesely SK, Chai-Adisaksopha C, et al. The impact of ruxolitinib on thrombosis in patients with polycythemia vera and myelofibrosis: a meta-analysis. Blood Coagul Fibrinolysis 2016; 27: 648-652. 